\title{
Molecular characteristics of carbapenem-resistant Enterobacteriaceae isolated from clinical infection and fecal survey samples in Southern China
}

\author{
Xiaonv Duan \\ Southern Medical University \\ Yingcheng Qin \\ Southern Medical University \\ Yuan Peng \\ Southern Medical University \\ YongYu Rui ( $\square$ yongyuruigroup@sina.com) \\ Southern Medical University
}

\author{
Research \\ Keywords: carbapenem-resistant Enterobacteriaceae, carbapenemase, mcr, KPC-2, fecal, molecular characteristics \\ Posted Date: August 7th, 2020 \\ DOI: https://doi.org/10.21203/rs.3.rs-52967/v1 \\ License: (c) (i) This work is licensed under a Creative Commons Attribution 4.0 International License. Read Full License
}




\begin{abstract}
Background: The identified rate of carbapenem-resistant Enterobacteriaceae (CRE) have been increasingly in the clinical infections. Here, a study of analysing the relationship of clinical infectious CRE and fecal carried CRE was performed.
\end{abstract}

Methods: Clinical CRE and fecal CRE were collected from hosiptal in China. Polymerase chain reaction (PCR)-based amplification and sequencing were performed to analyse the carriage of drug-resistant genes and mobile genetic elements (MGEs), Enterobacterial Repetitive Intergenic Consensus (ERIC) technology and whole genome sequencing (WGS) were used to analysis the characteristic of genetic structure of CRE isolates.

Results: 99 clinical CRE and 30 fecal CRE were collected, respectively. The top three strains present in the highest proportions were $K$. pneumoniae (86; $66.67 \%)$, E. cloacae (22; $17.05 \%)$, and E. coli $(11 ; 8.53 \%)$. Most of the isolates were susceptible to colistin ( $98.45 \%)$ and tigecycline $(98.45 \%)$. bla ${ }_{K P C}-2(96.03 \%)$ was the dominant carbapenemase gene in clinical CRE and fecal CRE, followed by bla ${ }_{N D M}(52.7 \%)$; co-existence of the $b / a_{K P C-2}$ and $b / a_{N D M}$ genes was detected in 63 (50.00\%) strains. One K. pneumoniae isolate co-producing NDM-5 and mcr-1, and one E. coli isolate co-producing KPC-2, IMP-4, and mcr-1 were detected. Two novel gene cassettes of intl2 were dectected. ERIC genotyping and genomic analysis revealed that $K$. pneumoniae isolated from clinical infections and fecal survey samples were same clone.

Conclusions: This is the first report of comparing the molecular characteristics of CRE isolated from clinical infection and fecal survey samples. we found that fecal carried CRE were closely related to CRE which caused infections.

\title{
Background
}

Carbapenem-resistant Enterobacteriaceae (CRE) have been listed by the World Health Organization (WHO) as high priority pathogens owing to their association with high mortality and morbidity ${ }^{[1]}$. CRE have spread rapidly around the world, posing great challenges to human health. The China Antimicrobial Resistance Surveillance Report (http://www.carss.cn/), the largest survey of antimicrobial resistance in China, reported that the rate of CRE is increasing every year; in particular, the rate of carbapenem-resistant $K$. pneumoniae increased from $4.9 \%$ in 2013 to $10.1 \%$ in 2018 . Carbapenem resistance in $K$. pneumoniae has been reported in all WHO regions and exceeded $50 \%$ of all isolates in two regions ${ }^{[2]}$. CRE can cause multiple infections, most frequently pneumonia, followed by urinary tract infections, primary bloodstream infections, skin and soft tissue infections, and surgical site infections ${ }^{[3]}$. Patients infected with CRE are at an increased risk of death due to the lack of appropriate antibiotics. CRE, especially carbapenem-resistant K. pneumoniae (CRKP), have a high potential to cause outbreaks in healthcare settings. Such outbreaks have been reported from several EU member states; e.g., the Czech Republic, France, Germany, Greece, Italy, Spain, the Netherlands, and the UK ${ }^{[4-10]}$. Therefore, the timely and accurate detection of CRE is important; investigating the mechanism of transmission and the route of drug resistance will also be necessary.

Intestinal flora forms a major reservoir of various bacteria, including multidrug resistant (MDR) bacteria, which serves as an important and large reservoir of resistance genes. It may greatly increase the transmission of resistance genes and the probability of nosocomial infections caused by MDR bacteria ${ }^{[11]}$. In particular, CRE can colonize the intestines of patients or individuals without underlying disease, greatly increasing the risk of clinical infection. Thus, studying the drug-resistant and molecular characteristics of CRE between clinical infection and fecal survey samples will also be important.

CRE can lead to clinical infection, which will increase the risk of standard antimicrobial therapy failing and increase the difficulty of treatment. CRE can also become part of the intestinal flora of healthy individuals who have not been exposed to antimicrobials. There is a risk of hidden transmission of CRE, and possibly even CRE outbreaks. The aim of this study was to compare the molecular characteristics of CRE isolated from clinical infection and fecal survey samples in Southern China, and provide information for the prevention and control of CRE infections.

\section{Methods}

\section{Bacterial isolates and species identification}

Between July 2016 and June 2019, 99 CRE were obtained from clinical infection samples (including sputum, puncture fluid, urine, instrument, and blood) collected from Nanfang Hospital, a large general and teaching hospital in Guangzhou, China.

A total of 2,000 fecal survey samples were collected from July 2016 to June 2019, followed by incubation on MacConkey agar plates with $2 \mu \mathrm{g} / \mathrm{mL}$ meropenem. All isolates were confirmed as Enterobacteriaceae by biochemical methods, and a total of $30 \mathrm{CRE}$ were collected. The study was approved by the Medical Ethics Committee of Nanfang Hospital Southern Medical University and conducted in compliance with the Declaration of Helsinki.

\section{Antimicrobial susceptibility testing}

Analysis of susceptibility to antimicrobial compounds was performed using the automated BD Phoenix 100 Microbiology System (Becton Dickinson and Co., Franklin Lakes, NJ, USA) according to the guidelines of the Clinical and Laboratory Standards Institute (CLSI) M100-S29 criteria. In addition, minimum inhibitory concentration (MIC) breakpoints of susceptibility to tigecycline were determined by E-test according to the guidelines of the European Committee on Antimicrobial Susceptibility Testing (EUCAST). E. coli ATCC 25922 and Pseudomonas aeruginosa ATCC 27853 were used as control strains for antimicrobial susceptibility testing.

\section{Molecular detection of carbapenemase genes}

The following carbapenemase-encoding genes were detected: the class A $\beta$-lactamase gene blaKPC ${ }^{12]}$; class $\mathrm{B}$ metallo- $\beta$-lactamase genes: blaIMP[12], blaVIM ${ }^{[12]}$, blaSPM ${ }^{[12]}$, and blaNDM ${ }^{[12]}$; and class D oxacillinase genes: blaOXA-48-like ${ }^{[12]}$. The common ESBL genes CTX-M[13], SHM ${ }^{[13]}$, and TEM ${ }^{[13]}$ were also 
detected.

\section{Molecular detection of colistin-related resistance genes}

The colistin-related resistance genes $m c r-7^{[14]}, m c r-2^{[14]}, m c r-3^{[14]}, m c r-4^{[14]}$, and $m c r-5^{[14]}$ were detected. There are substantial differences in the nucleotide sequences of the five subtypes of the morgene, so each subtype had a specific pair of primers.

\section{Detection of MGEs}

MGE-related resistance genes, including integrons (int|1 ${ }^{[15,16]}$, int| $2^{[15,17]}$, and int|3 ${ }^{[15]}$ ) and ISCRs ${ }^{[18]}$, including the variable region of class 1 integron, class 2 integron, and ISCR1, were also analyzed.

The primers including the IncFIl plasmid replicon ${ }^{[19]}$ used for PCR are shown in Supplementary Table 1.

\section{Whole genome sequencing and analysis}

Three KPC-producing K. pneumoniae 156070, 158590, and NFYY0065 were submitted for WGS. The whole genome was sequenced using the Single Molecule Real-Time sequencing platform with the PacBio sequencer and Illumina HiSeq at the Health Time Gene Institute (Shenzhen, China).

\section{ERIC-PCR}

ERIC-PCR was performed on all isolates depending on the species ${ }^{[20]}$. Band comparisons were performed by clustering analysis based on the unweighted pairgroup method with arithmetic mean of isolates using NTSYS.

\section{Results \\ Identification of isolates}

During the study period, clinical infection samples were collected from 20 departments of Nanfang hospital; the top four departments were hematology (37.4\%), intensive care unit (ICU) (22.2\%), rehabilitation (7.1\%), and the surgical department (4.0\%). A total of 99 CRE from clinical infection samples and 30 CRE from fecal survey samples were isolated. There was no obvious difference in the types of CRE between clinical and fecal samples. The species present in the highest proportions among the six different species isolated from clinical infection samples were K. pneumoniae (73.74\%), E. cloacae (15.15\%), and E. coli (7.07\%); the species present in the highest proportions among the seven different species isolated from fecal survey samples were $K$. pneumoniae (43.33\%), E. cloacae (23.33\%), and E. coli (13.33\%) (Table 1).

\section{Antimicrobial susceptibility testing}

According to the MIC values of tested antibiotics (Supplementary Tables 2 and 3). All isolates were resistant to cephems and carbapenems, and most of the isolates were sensitive to colistin and tigecycline. Among clinical CRE, the sensitivities to amikacin, tetracycline, and sulfamethoxazole were $35.35 \%$, $35.35 \%$, and $32.32 \%$, respectively. Among fecal CRE, the sensitivities to amikacin, tetracycline, and sulfamethoxazole were $56.67 \%, 36.67 \%$, and $40.00 \%$, respectively. We found that the sensitivities of different species to amikacin and tetracycline vary widely, and CRE isolated from feces were generally more sensitive to antibiotics than clinical strains with the exception of $K$. pneumoniae (Table 2).

\section{Carbapenemase-encoding genes}

Strains with carbapenemase genes accounted for 126 (97.67\%) of all the isolates; 98 (98.99\%) CRE isolated from clinical samples and 28 (93.33\%) CRE isolated from fecal samples were positive for carbapenemase genes. Among isolates containing carbapenemase genes, the most prominent carbapenemase gene was blaKPC-2 $(121 ; 93.8 \%)$ and blaNDM was the second most frequent (68 isolates, $52.7 \%)$. blaNDM-1 and blaNDM-5 were detected in 40 clinical strains and 11 clinical strains, respectively, and fecal strains carried four types of blaNDM genes: blaNDM-1 (11; 39.29\%), blaNDM-5 (5; 17.86\%), and blaNDM-4 (1; $3.57 \%$ ). Co-existence of the blaKPC and blaNDM genes was detected in 47 clinical strains, and co-existence of the blaKPC and blaNDM genes was detected in 15 fecal strains. It is notable that 20 strains simultaneously carried the blaKPC genes and two types of metallo- $\beta$-lactamases genes, including 17 clinical and 3 fecal isolates. For other carbapenemase genes in all strains, 38 (29.46\%) isolates were positive for blaIMP, 4 (3.10\%) for blaVIM, and 125 (96.90\%) for ESBL genes. $m c r-1$ was only detected in fecal CRE, including one $K$. pneumoniae isolate co-producing NDM-5 and mcr-1, and one E. coli isolate co-producing KPC-2, IMP-4, and mcr-1. blaOXA-48 and blaSPM were not identified (Table 3).

\section{MGE-related resistance genes}

Class I and II integrons and ISCR1 were detected in 101 (78.29\%), 6 (4.65\%), and 90 (69.77\%) strains, respectively, while class III integrons were absent. Class I and II integrons and ISCR1 were detected in 77 (77.77\%), 2 (2.02\%), and 71 (71.71\%) strains isolated from clinical samples, and class I and II integrons and ISCR1 were detected in 24 (80.00\%), 4 (13.33\%), and 19 (63.33\%) strains isolated from feces, respectively. The prevalence of intl1, intl2, and ISCR1 varied in different species (Supplementary Table 4); the gene cassettes of intl1, intI2, and ISCR1 were detected in 29 (28.71\%), 3 (50\%), and 4 (4.44\%), respectively. Sequencing results revealed that the variant region of class 1 integron has eight distinct gene cassettes, and the variant region of the class 2 integron had two novel gene cassettes (dfrA1-sat2-aadA1-ybeA-ybfA-ybfB-ybgA-ORF(5)-Tra,dfrA1-sat2-aadA1-ybeA-ybfA-ybfB-ybgA-IS3-IS110-IS3-Tra-ybgA) (Table 4).

\section{Replicon of IncFIl plasmids}


Most of the strains $(60.5 \%, 78 / 129)$ were positive for the replicon of IncFll plasmids. In the clinical and fecal strains, the IncFIl replicon was observed in $69.23 \%(54 / 78)$ and $30.77 \%$ (24/78) of strains, and the IncFIl replicon $(84.62 \%, 66 / 78)$ was carried from K. pneumoniae.

\section{Comparative genomic analysis of two KPC-CRKP}

Using WGS, we determined that the complete genome of 156070-KPC-CRKP consists of a 5,415,677 bp circular chromosome (chr156070) and a 138,959 bp plasmid (p156070-KPC). The complete genome of 158590-KPC-CRKP consisted of a 5,439,711 bp circular chromosome (chr158590), a 138,959 bp plasmid (p158590-KPC), and a 10,060 bp plasmid (data not shown). Both 156070-KPC-CRKP and 158590-KPC-CRKP contained 89 drug-resistant genes, and there were four genes related to $\beta$-lactamase antibiotic resistance: SHV-11, KPC-2, CTX-M-65, and TEM-1b.

The complete sequence analysis showed that chr 156070 contained 5227 genes with a G + C content of $57.40 \%$, and chr 158590 contained 5262 genes with a $\mathrm{G}+\mathrm{C}$ content of $57.37 \%$. Both chr156070 and chr158590 contained blaSHV-11. BLAST analysis revealed that similarities in the chromosomes of the two KPCCRKP were greater than $95 \%$. Comparative genomic analysis showed that the core genes of chr156070 and chr158590 accounted for $89 \%$ of all genes, and chr156070 had no specific resistant genes compared with chr158590. Co-linear analysis found that although there were structural differences between chr156070 and chr158590, they had a high degree of similarity and high coverage for one another (Fig. 1). They are ST11 by multilocus sequence typing. chr156070 and chr158590 were similar. BLASTn analysis revealed that chr156070 and chr158590 were highly similar to $18 \mathrm{CPO} 060$ and KPNIH45, and phylogenetic analysis showed that chr156070 and chr158590 had a close evolutionary relationship and high affinity to KPNIH45, which was derived from CRKP isolated from a US hospital in 2018.

The $\mathrm{p} 156070-\mathrm{KPC}$ is a circular plasmid containing 184 putative open reading frames (ORFs) with a G + C content of 53.95\%, and p158590-KPC was a circular plasmid containing 184 putative ORFs with a G + C content of 53.95\%. Both p156070-KPC and p158590-KPC had the same replication protein RepA1 of IncFII plasmids. The p156070-KPC had one unique gene relative to p158590-KPC, and p158590-KPC had two unique genes relative to $\mathrm{p} 156070-\mathrm{KPC}$. Both $\mathrm{p} 156070-$ KPC and p158590-KPC co-carried the blaKPC-2, blaCTX-M-65, and blaTEM-1b genes. BLAST analysis showed that p156070-KPC was highly similar to p158590-KPC at the nucleotide level (100\% identities and 100\% query coverage), and colinearity analysis revealed that the plasmid was divided into three parts: the first segment plus chain of p156070-KPC and p158590-KPC were performed collinear comparison from 1 bp to 32195 bp, with a similarity of $99 \%$; the second segment of p156070-KPC and p158590-KPC plasmid were performed translocation comparison from 31,374 bp to 106,077 bp, with a similarity of $100 \%$, the phenomenon might be result from the rearrangement of small fragments that maked up the A fragment and B fragment due to MGEs; the third segment 105,256 bp to 138,959 bp of p156070-KPC and p158590-KPC were collinear comparison, with a similarity of 100\% (Fig. 2).

The resistant gene structure of the two plasmids showed that blaKPC-2 and blaCTX-M-65 existed in the prophage sequence, with surrounding MGEs, which suggested that the blaKPC-2 and blaCTX-M-65 genes of the two plasmids were from the insertion of foreign genes (Supplementary Fig. 1). BLASTN analysis showed that p156070-KPC and p158590-KPC were 96\% similar to the previously reported IncFll plasmid p283747-KPC (MF168406.1), which harbored blaKPC2 between ISKpn27 and ISKpn6 (Fig. 3), the structures of blaCTX-M-65 and blaTEM-1 were basically identical to pKSH203-KPC (CP034324.1).

Phylogenetic analysis showed that p156070-KPC and p158590-KPC are closely genetically related to most of the IncFIl plasmids previously reported (Fig. 4). The 17 IncFII plasmids were selected from the database, and the pNFYYOY11 plasmids were the IncFIl plasmids carrying the blaKPC gene sequenced by us (Supplementary Table 5).

\section{ERIC-PCR}

Genotyping by ERIC-PCR demonstrated greater than $90 \%$ similarity of Klebsiella pneumoniae in different samples, including clinical infection and fecal survey samples (Supplementary Fig. 2), suggesting they may have originated from the same clone. However, E. cloacae and E. coli detected in clinical and fecal samples had a high genetic diversity.

\section{Discussion}

In all isolates, the top three species were K. pneumoniae (66.67\%), E. cloacae (17.05\%), and E. coli (8.53\%); thus, CRKP was more abundant than other species This is consistent with Spiliopoulon et al. ${ }^{[21]}$, which showed that $K$. pneumoniae was present in 1,137 (77.9\%) of 1,460 clinical infectious samples of hospitalized patients around the world between 2015 and 2017. The number of clinical infections caused by $K$. pneumoniae is increasing; in particular, the number of CRKP is increasing ${ }^{[22]}$. In our study, the species present in the highest proportions in fecal survey samples were also $K$. pneumoniae, E. cloacae, and E. coli. The fecal samples were primarily obtained from individuals who had not previously used related antibiotics; however, they carried a high proportion of CRE similar to clinical infection samples, indicating that CRE can also exist in the intestines of normal individuals without a history of antibiotic use and that fecal CRE may be associated with CRE causing clinical infection.

The sensitivity of CRE to antibiotics has reduced, which is a challenge for clinical treatment. In our study, clinical and fecal CRE were resistant to most of the antibiotics tested and only showed a high sensitivity to tigecycline and colistin. The susceptibilities of clinical CRE to tigecycline and colistin were $100 \%$ and $100 \%$, and the susceptibilities of fecal CRE were $93.33 \%$ and $93.33 \%$, respectively. Spiliopoulon et al. ${ }^{[21]}$ reported that the susceptibilities of meropenem-nonsusceptible Enterobacteriaceae to colistin and tigecycline were $92.4 \%$ and $77.4 \%$, respectively; colistin and tigecycline were shown to be active against MBLpositive isolates (susceptibilities of $92.1 \%$ and $71.9 \%$, respectively) in Asia. Wang et al. ${ }^{[23]}$ reported that 1801 CRE isolates showed high susceptibility to colistin (96.9\%), followed by tigecycline (89.7\%). Thus, CRE are still highly sensitive to colistin and tigecycline; in support of this, we found that colistin has high activity against CRE in Southern China, which will help in the choice of treatment of clinical infections. The susceptibility of different CRE species to amikacin varied, with a lower rate in K. pneumoniae (24.4\%) than in E. coli and E. cloacae (> $72 \%)$. This result provides important data for selecting specific drug and aminoglycoside combinations for empiric therapy of infections caused by these species. 
CRE is resistant to carbapenem antibiotics for different reasons, but the high rate of carbapenemase genes detected in this study is likely the primary mechanism. Our data showed that the prevalent carbapenemases in Southern China primarily encoded KPC and NDM, and especially KPC, which may be related to a high proportion of CRKP. Notably, blaKPC is also commonly found in other species. KPC has already spread widely to different species, mainly KPC-2 in Southern China. We found a similar proportion of blaKPC between clinical and fecal CRE, especially CRKP, indicating that fecal KPC-CRKP may be closely related to KPC-CRKP isolated from clinical infection samples. In addition, we found that one $K$. pneumoniae isolate contained blaNDM-5 and mcr-1, and one E. coli isolate contained blaKPC-2, blaIMP-4, and $m c r-1$, which have not been reported previously. The mcr and carbapenemases-positive strains were isolated from fecal survey samples from individuals who had not previously used carbapenem and colistin, indicating that $m c r-1$ and carbapenemase genes may also be present in the gastrointestinal tract of patients who have not used related antibiotics. If individuals expressing resistance genes have infections, it will lead to the failure of antibiotic treatment, and increase the risk of transmission to other patients. Therefore, strengthening the monitoring of feces is necessary to prevent the spread of drug-resistant bacteria and drug-resistant genes.

Our data showed that clinical and fecal CRE carry MGE-related genes, which is consistent with the high prevalence of carbapenemase genes. Carbapenemaseproducing CRE strains carry resistance genes on mobile plasmids or MGEs that can shuttle between resistant and susceptible strains ${ }^{[24,25]}$, especially those encoding KPC and NDM. This suggested that MGEs were important for the spread of carbapenemase genes and multidrug resistance of strains. In addition, we identified two novel gene cassette structures of class II integrons, which have not been previously reported.

We found a high genetic diversity of E. cloacae and $E$. coli in different samples by ERIC genotyping; however, K. pneumoniae detected in clinical and fecal samples showed greater than $90 \%$ similarity, indicating that fecal CRE were closely related to CRE isolated from clinical infection samples, and that they may have originated from the same clone. Clinical and fecal samples were obtained from different places in South China, suggesting that KPC-CRKP has spread widely. However, all strains were isolated from samples collected between 2016 and 2019, indicating that there was no outbreak in the short term.

\section{Conclusions}

This was the first study to compare the molecular characteristics of CRE isolated from clinical infection and fecal survey samples in Southern China. We found that CRKP isolated from fecal survey samples were closely related to CRKP isolated from clinical infections samples. CRE can spread between human, animals and the environment by fecal contamination and especially outbreak. Thus, Strengthening the monitoring and management of feces is essential for the prevention and control of CRE.

\section{Abbreviations}

CRE carbapenem-resistant Enterobacteriaceae

CRKP carbapenem-resistant Klebsiella pneumoniae

MDR multidrug-resistant

ERIC enterobacterial repetitive intergenic consensus

WGS whole genome sequencing

\section{Declarations}

\section{Ethics approval and consent to participate:}

Verbal informed consent was obtained from all participants rather than written consent for personal reasons. The samples used were the specimens remaining after the patient's clinical examination. The study was approved by the Medical Ethics Committee of Nanfang Hospital Southern Medical University and conducted in compliance with the Declaration of Helsinki (No. NFEC-2014-002).

\section{Consent to publish:}

Not applicable.

\section{Availability of data and materials:}

All data generated or analyzed in this study are included in this published article and its supplementary information files.

\section{Competing interests:}

The authors declare that they have no competing interests.

Funding: 
This study was supported by grants from Guangdong Province Science and Technology Project (Nos. 2014A010107011 and 2015A020211011) and Guangzhou City Science and Technology (No. 201510010167). The funding body provides financial support in designing research and collecting, analyzing, interpreting data and writing articles.

\section{Authors' contributions:}

DXN carried out the experiments, data organization and analysis, and contributed to writing the manuscript and annotating the results. QYC collected all of the isolates and clinical data. PY participated in a subset of the experiments. RYY contributed to the design of the study and assisted in the drafting of the manuscript. All authors have read and approved the manuscript.

\section{Acknowledgments:}

We thank International Science Editing.

\section{References}

1. Kopotsa K, Osei Sekyere J, Mbelle NM. Plasmid evolution in carbapenemase-producing Enterobacteriaceae: a review. Ann Ny Acad Sci. 2019;1457:61-91.

2. World Health Organization. Antimicrobial Resistance Global Report on Surveillance. Geneva: WHO; 2014. Available from:

3. https://apps.who.int/iris/bitstream/handle/10665/112642/9789241564748_eng.pdf?sequence=1.

4. Tischendorf J, de Avila RA, Safdar N. Risk of infection following colonization with carbapenem-resistant Enterobactericeae: A systematic review. Am J Infect Control. 2016;44:539-43.

5. Vergara-Lopez S, Dominguez MC, Conejo MC, Pascual A, Rodriguez-Bano J. Lessons from an outbreak of metallo-beta-lactamase-producing Klebsiella oxytoca in an intensive care unit: the importance of time at risk and combination therapy. J Hosp Infect. 2015;89:123-31.

6. Voulgari E, Gartzonika C, Vrioni G, et al. The Balkan region: NDM-1-producing Klebsiella pneumoniae ST11 clonal strain causing outbreaks in Greece. J Antimicrob Chemother. 2014;69:2091-7.

7. Hrabak J, Papagiannitsis CC, Studentova V, Jakubu V, Fridrichova M, Zemlickova H. Carbapenemase-producing Klebsiella pneumoniae in the Czech Republic in 2011. Euro Surveill. 2013;18:20626.

8. Zweigner J, Gastmeier P, Kola A, Klefisch FR, Schweizer C, Hummel M. A carbapenem-resistant Klebsiella pneumoniae outbreak following bronchoscopy. Am J Infect Control. 2014;42:936-7.

9. Gharbi M, Moore LS, Gilchrist M, et al. Forecasting carbapenem resistance from antimicrobial consumption surveillance: Lessons learnt from an OXA-48producing Klebsiella pneumoniae outbreak in a West London renal unit. Int J Antimicrob Agents. 2015;46:150-6.

10. Gaibani P, Colombo R, Arghittu M, et al. Successful containment and infection control of a Carbapenem-resistant Klebsiella pneumoniae outbreak in an Italian hospital. New Microbiol. 2014;37:87-90.

11. Dautzenberg MJ, Ossewaarde JM, de Kraker ME, et al. Successful control of a hospital-wide outbreak of OXA-48 producing Enterobacteriaceae in the Netherlands, 2009 to 2011. Euro Surveill 2014;19.

12. Li S, Duan X, Peng Y, Rui Y. Molecular characteristics of carbapenem-resistant Acinetobacter spp. from clinical infection samples and fecal survey samples in Southern China. Bmc Infect Dis. 2019;19:900.

13. Poirel L, Walsh TR, Cuvillier V, Nordmann P. Multiplex PCR for detection of acquired carbapenemase genes. Diagn Microbiol Infect Dis. 2011;70:119-23.

14. Bubpamala J, Khuntayaporn P, Thirapanmethee K, Montakantikul P, Santanirand P, Chomnawang MT. Phenotypic and genotypic characterizations of extended-spectrum beta-lactamase-producing Escherichia coli in Thailand. Infect Drug Resist. 2018;11:2151-7.

15. Rebelo AR, Bortolaia V, Kjeldgaard JS, et al. Multiplex PCR for detection of plasmid-mediated colistin resistance determinants, mcr-1, mcr-2, mcr-3, mcr-4 and mcr-5 for surveillance purposes. Euro surveillance: bulletin Europeen sur les maladies transmissibles = European. communicable disease bulletin 2018;23:11-29.

16. Shibata N, Doi Y, Yamane K, et al. PCR typing of genetic determinants for metallo-beta-lactamases and integrases carried by gram-negative bacteria isolated in Japan, with focus on the class 3 integron. J Clin Microbiol. 2003;41:5407-13.

17. Skurnik D, Le Menac'H A, Zurakowski D, et al. Integron-associated antibiotic resistance and phylogenetic grouping of Escherichia coli isolates from healthy subjects free of recent antibiotic exposure. Antimicrob Agents Chemother. 2005;49:3062-5.

18. White PA, Mclver CJ, Rawlinson WD. Integrons and gene cassettes in the enterobacteriaceae. Antimicrob Agents Chemother. 2001;45:2658-61.

19. Rui Y, Lu W, Li S, Cheng C, Sun J, Yang Q. Integrons and insertion sequence common region 1 (ISCR1) of carbapenem-non-susceptible Gram-negative bacilli in fecal specimens from 5000 patients in southern China. Int J Antimicrob Agents. 2018;52:571-6.

20. Villa L, García-Fernández A, Fortini D, Carattoli A. Replicon sequence typing of IncF plasmids carrying virulence and resistance determinants. J Antimicrob Chemoth. 2010;65:2518-29.

21. Versalovic J, Koeuth T, Lupski JR. Distribution of repetitive DNA sequences in eubacteria and application to fingerprinting of bacterial genomes. Nucleic Acids Res. 1991;19:6823-31.

22. Spiliopoulou I, Kazmierczak K, Stone GG. In vitro activity of ceftazidime/avibactam against isolates of carbapenem-non-susceptible Enterobacteriaceae collected during the INFORM global surveillance programme (2015-17). J Antimicrob Chemoth 2019.

Page 6/11 
23. Shu L, Lu Q, Sun R, et al. Prevalence and phenotypic characterization of carbapenem-resistant < em > Klebsiella pneumoniae $<$ /em > strains recovered from sputum and fecal samples of ICU patients in Zhejiang Province, China. 2019;Volume 12:11-18.

24. Wang Q, Wang X, Wang J, et al. Phenotypic and Genotypic Characterization of Carbapenem-resistantEnterobacteriaceae: Data From a Longitudinal Largescale CRE Study in China (2012-2016). Clin Infect Dis. 2018;67:196-205.

25. Mathers AJ, Peirano G, Pitout JD. The role of epidemic resistance plasmids and international high-risk clones in the spread of multidrug-resistant Enterobacteriaceae. Clin Microbiol Rev. 2015;28:565-91.

26. Mathers AJ, Cox HL, Kitchel B, et al. Molecular dissection of an outbreak of carbapenem-resistant enterobacteriaceae reveals Intergenus KPC carbapenemase transmission through a promiscuous plasmid. Mbio. 2011;2:e204-11.

\section{Tables}

\section{Table 1 Numbers of isolated Carbapenem-resistant Enterobacteriaceae}

\begin{tabular}{|c|c|c|c|}
\hline Species & clinical samples & fecal samples & Total \\
\hline K.peumoniae & 73(73.74) & 13(43.33) & $86(66.67)$ \\
\hline K.oxytoca & $2(2.02)$ & $2(6.67)$ & $4(3.10)$ \\
\hline E.coli & $7(7.07)$ & $4(13.33)$ & $11(8.53)$ \\
\hline \multirow[t]{2}{*}{$\begin{array}{l}\text { E. aerogenes } \\
\text { E.cloacac }\end{array}$} & 15(15.15) & $7(23.33)$ & $22(17.05)$ \\
\hline & $1(1.01)$ & $1(3.33)$ & $2(1.55)$ \\
\hline E.asburiac & $1(1.01)$ & $0(0.00)$ & $1(0.78)$ \\
\hline C.freundii & $0(0.00)$ & 1(3.33) & $1(0.78)$ \\
\hline S.marcescens & $0(0.00)$ & $2(6.67)$ & $2(1.55)$ \\
\hline Total & 99 & 30 & 129 \\
\hline
\end{tabular}

\section{Table 2 Antimicrobial susceptibility testing of Carbapenem-resistant Enterobacteriaceae}

\begin{tabular}{|c|c|c|c|c|c|c|c|c|c|}
\hline & \multicolumn{9}{|c|}{ No.(\%) of isolates: } \\
\hline & \multicolumn{4}{|l|}{ clinical samples } & \multicolumn{4}{|l|}{ fecal samples } & \multirow{2}{*}{$\begin{array}{l}\text { Total } \\
\text { Enter } \\
(n=1 ;\end{array}$} \\
\hline & $\begin{array}{l}\text { K.pneumoniae } \\
(n=73)\end{array}$ & $\begin{array}{l}\text { E.cloacae } \\
(n=15)\end{array}$ & $\begin{array}{l}\text { E.coli } \\
(n=7)\end{array}$ & $\begin{array}{l}\text { Total* } \\
(n=99)\end{array}$ & $\begin{array}{l}\text { K.pneumoniae } \\
(n=13)\end{array}$ & $\begin{array}{l}\text { E.cloacae } \\
(n=7)\end{array}$ & $\begin{array}{l}\text { E.coli } \\
(n=4)\end{array}$ & $\begin{array}{l}\text { Total* } \\
(n=30)\end{array}$ & \\
\hline \multicolumn{10}{|l|}{ Antimicrobial agent } \\
\hline Aztreonam & $5(6.85)$ & $3(20.00)$ & $2(28.57)$ & $10(10.10)$ & $1(7.69)$ & $2(28.57)$ & $2(50.00)$ & $6(20.00)$ & $16(12$ \\
\hline Amikacin & $18(24.66)$ & $12(80.00)$ & $4(57.14)$ & $35(35.35)$ & $3(23.08)$ & $6(85.71)$ & $4(100)$ & $17(56.67)$ & $52(4 \mathrm{C}$ \\
\hline Imipenem & $0(0.00)$ & $0(0.00)$ & $0(0.00)$ & $0(0.00)$ & $0(0.00)$ & $0(0.00)$ & $0(0.00)$ & $0(0.00)$ & $0(0.0$ \\
\hline Meropenem & $0(0.00)$ & $0(0.00)$ & $0(0.00)$ & $0(0.00)$ & $0(0.00)$ & $0(0.00)$ & $0(0.00)$ & $0(0.00)$ & $0(0.0$ \\
\hline${ }^{\mathrm{a} C}$ Colistin & $73(100)$ & $15(100)$ & $7(100)$ & $99(100)$ & $12(92.31)$ & $7(100)$ & $3(75.00)$ & 28(93.33) & $127(\stackrel{c}{c}$ \\
\hline Levofloxacin & $3(4.11)$ & $1(6.67)$ & $0(0.00)$ & $4(4.04)$ & $1(7.69)$ & $1(14.29)$ & $0(0.00)$ & $5(16.67)$ & $9(6.9$ \\
\hline Ciprofloksacin & $1(1.37)$ & $1(6.67)$ & $0(0.00)$ & $2(2.02)$ & $0(0.00)$ & $1(14.29)$ & $1(25.00)$ & $2(6.67)$ & $4(3.1$ \\
\hline Tetracycline & $27(36.99)$ & $5(33.33)$ & $0(0.00)$ & $35(35.35)$ & $4(30.77)$ & $3(42.86)$ & $1(25.00)$ & $11(36.67)$ & $46(35$ \\
\hline $\begin{array}{l}\text { Trimethoprim- } \\
\text { Sulfamethoxazole }\end{array}$ & $25(34.25)$ & $5(33.33)$ & $0(0.00)$ & $32(32.32)$ & $6(46.15)$ & $2(28.57)$ & $1(25.00)$ & $12(40.00)$ & $44(3<$ \\
\hline${ }^{\mathrm{b}}$ Tigecycline & 73(100) & $15(100)$ & $7(100)$ & $99(100)$ & $11(84.62)$ & $7(100)$ & $4(100)$ & 28(93.33) & $127(\stackrel{c}{-}$ \\
\hline
\end{tabular}


*shows the number of all isolates which are susceptibility to antimicrobial compounds, only three kinds of Carbapenem-resistant Enterobacteriaceae with the highest separation ratios are shown in the table.

${ }^{\text {a }}$ Antimicrobial susceptibility to colistin according to Clinical and Laboratory Standards Institute (CLSI) M100-S29 criteria

${ }^{\mathrm{b}}$ Antimicrobia susceptibility to tigecycline according to European Committee on Antimicrobial Susceptibility Testing (EUCAST).

\section{Table 3 Carbapenemase-encoding genes and colistin-related resistance gene of Carbapenem-resistant Enterobacteriaceae}

\begin{tabular}{|c|c|c|c|c|c|c|c|c|c|}
\hline & \multicolumn{9}{|c|}{ No.(\%) of isolates: } \\
\hline & \multicolumn{4}{|l|}{ clinical samples } & \multicolumn{4}{|l|}{ fecal samples } & \multirow{3}{*}{$\begin{array}{l}\text { Total } \\
\text { Enter } \\
(n=1 ;\end{array}$} \\
\hline & K.pneumoniae & E.cloacae & E.coli & Total* & K.pneumoniae & E.cloacae & E.coli & Total* & \\
\hline & $(n=73)$ & $(n=15)$ & $(n=7)$ & $(n=99)$ & $(n=13)$ & $(n=7)$ & $(n=4)$ & $(n=30)$ & \\
\hline \multicolumn{10}{|c|}{ Carbapenemase-encoding genes } \\
\hline \multicolumn{10}{|c|}{ class A $\beta$-lactamases } \\
\hline blaKPC & $66(90.41)$ & 14(93.33) & $7(100)$ & $94(94.94)$ & $12(92.31)$ & $5(71.43)$ & $4(100)$ & $27(90.00)$ & $121(\mathrm{c}$ \\
\hline \multicolumn{10}{|c|}{$\begin{array}{l}\text { class B metallob- } \beta \text { - } \\
\text { lactamases }\end{array}$} \\
\hline blalMP & $18(24.66)$ & $6(40.00)$ & $2(28.57)$ & $29(29.29)$ & $3(23.08)$ & $2(28.57)$ & $2(50.00)$ & $9(30.00)$ & $38(2 \varsigma$ \\
\hline blaVIM & $2(2.74)$ & $2(13.33)$ & $0(0.00)$ & $4(4.04)$ & $0(0.00)$ & $0(0.00)$ & $0(0.00)$ & $0(0.00)$ & $4(3.1)$ \\
\hline blaSPM & $0(0.00)$ & $0(0.00)$ & $0(0.00)$ & $0(0.00)$ & $0(0.00)$ & $0(0.00)$ & $0(0.00)$ & $0(0.00)$ & $0(0.0$ \\
\hline blaNDM & $35(47.95)$ & $7(46.67)$ & $5(71.43)$ & $51(51.51)$ & $11(84.62)$ & $2(28.57)$ & $2(50.00)$ & $17(56.67)$ & $68(52$ \\
\hline \multicolumn{10}{|c|}{ class D oxacillinases } \\
\hline blaOXA-48-like & $0(0.00)$ & $0(0.00)$ & $0(0.00)$ & $0(0.00)$ & $0(0.00)$ & $0(0.00)$ & $0(0.00)$ & $0(0.00)$ & $0(0.0$ \\
\hline \multicolumn{10}{|l|}{ ESBL genes } \\
\hline blactX-M & $18(24.66)$ & $1(6.67)$ & $3(42.86)$ & $24(24.24)$ & $4(30.77)$ & $2(28.57)$ & $1(25.00)$ & $9(30.00)$ & $33(25$ \\
\hline blaTEM & $59(80.82)$ & $10(66.67)$ & $6(85.71)$ & 78(78.79) & $11(84.62)$ & $7(100)$ & $4(100)$ & $27(90.00)$ & 105(ع \\
\hline blaSHV & 68(93.15) & 11(73.33) & $5(71.43)$ & $87(87.88)$ & $11(84.62)$ & $6(85.71)$ & $4(100)$ & $26(86.67)$ & 113(ع \\
\hline \multicolumn{10}{|c|}{$\begin{array}{l}\text { colistin-related resistance } \\
\text { gene }\end{array}$} \\
\hline$m c r-1$ & $0(0.00)$ & $0(0.00)$ & $0(0.00)$ & $0(0.00)$ & $1(7.69)$ & $0(0.00)$ & $1(25.00)$ & $2(6.67)$ & $2(1.5$ \\
\hline$m c r-2$ & $0(0.00)$ & $0(0.00)$ & $0(0.00)$ & $0(0.00)$ & $0(0.00)$ & $0(0.00)$ & $0(0.00)$ & $0(0.00)$ & $0(0.0$ \\
\hline$m c r-3$ & $0(0.00)$ & $0(0.00)$ & $0(0.00)$ & $0(0.00)$ & $0(0.00)$ & $0(0.00)$ & $0(0.00)$ & $0(0.00)$ & $0(0.0$ \\
\hline$m c r-4$ & $0(0.00)$ & $0(0.00)$ & $0(0.00)$ & $0(0.00)$ & $0(0.00)$ & $0(0.00)$ & $0(0.00)$ & $0(0.00)$ & $0(0.0$ \\
\hline$m c r-5$ & $0(0.00)$ & $0(0.00)$ & $0(0.00)$ & $0(0.00)$ & $0(0.00)$ & $0(0.00)$ & $0(0.00)$ & $0(0.00)$ & $0(0.0$ \\
\hline
\end{tabular}

*shows the number of all isolates which are susceptibility to antimicrobial compounds, only three kinds of Carbapenem-resistant Enterobacteriaceae with the highest separation ratios are shown in the table.

\section{Table 4 Characterization of integrons and ISCR in Carbapenem-resistant Enterobacteriaceae}




\begin{tabular}{|c|c|c|}
\hline Mobile genetic elements & Gene cassette array & No.(\%) of isolates ${ }^{a}$ \\
\hline \multirow[t]{8}{*}{ int/1 } & aadB-aadA2 & $1(3.12)$ \\
\hline & dfrA1-aadA1 & $9(28.12)$ \\
\hline & dfrA17-aadA5 & $1(3.12)$ \\
\hline & arr-3-dfrA27 & $2(6.25)$ \\
\hline & aadA8b-orfF-dhfrxll & $3(9.37)$ \\
\hline & ORFIII-ORFII-orfF-dfrA12 & $4(12.50)$ \\
\hline & aadA3c-orfF-dfrA12 & $1(3.12)$ \\
\hline & aadA2-orfF-dfrA12 & $8(25.00)$ \\
\hline \multirow[t]{2}{*}{ int/2 } & dfrA1-sat2-aadA1-ybeA-ybfA-ybfB-ybgA-ORF(5)-Tra & $2(6.25)$ \\
\hline & dfrA1-sat2-aadA1-ybeA-ybfA-ybfB-ybgA-IS3-IS110-IS3-Tra-ybgA & $1(3.12)$ \\
\hline \multirow[t]{4}{*}{ ISCR1 } & qnrB6-qacE & $1(25.00)$ \\
\hline & SapA-qnrB91-qacE & $1(25.00)$ \\
\hline & qnrB19-qacE & $1(25.00)$ \\
\hline & IS630-hypA-qacE & $1(25.00)$ \\
\hline
\end{tabular}

\section{Figures}

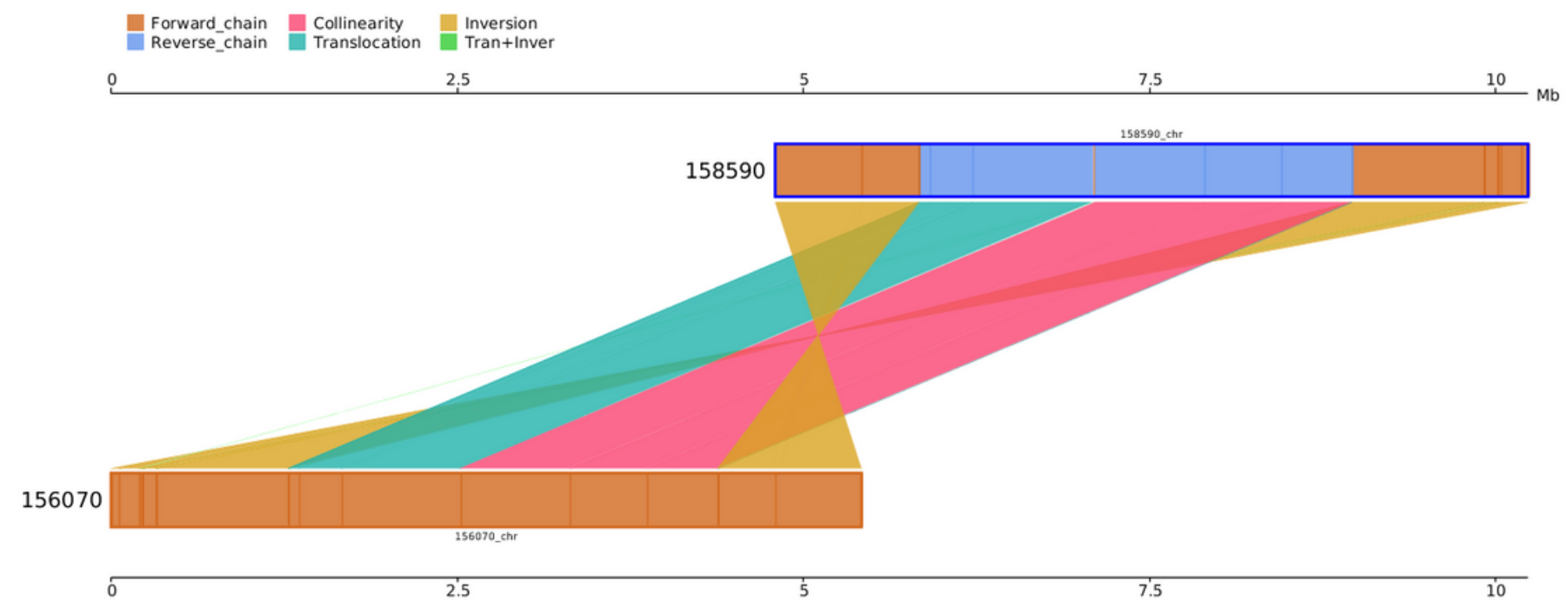

\section{Figure 1}

Co-linear analysis found that although there were structural differences between chr156070 and chr158590, they had a high degree of similarity and high coverage for one another. 


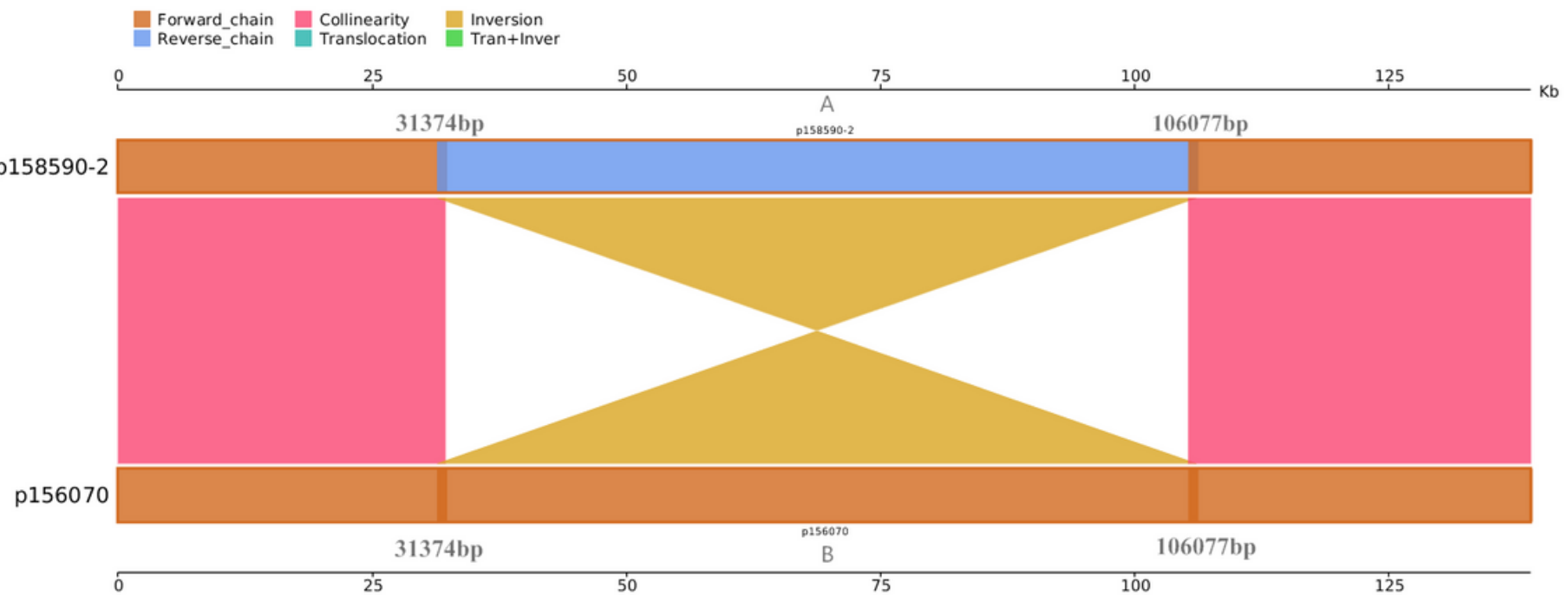

\section{Figure 2}

The $\mathrm{p} 156070-\mathrm{KPC}$ had one unique gene relative to $\mathrm{p} 158590-\mathrm{KPC}$, and $\mathrm{p} 158590-\mathrm{KPC}$ had two unique genes relative to $\mathrm{p} 156070-\mathrm{KPC}$. Both $\mathrm{p} 156070-\mathrm{KPC}$ and p158590-KPC co-carried the blaKPC-2, blaCTX-M-65, and blaTEM-1b genes. BLAST analysis showed that p156070-KPC was highly similar to p158590-KPC at the nucleotide level (100\% identities and $100 \%$ query coverage), and colinearity analysis revealed that the plasmid was divided into three parts: the first segment plus chain of p156070-KPC and p158590-KPC were performed collinear comparison from $1 \mathrm{bp}$ to $32195 \mathrm{bp}$, with a similarity of $99 \%$; the second segment of p156070-KPC and p158590-KPC plasmid were performed translocation comparison from 31,374 bp to 106,077 bp, with a similarity of $100 \%$, the phenomenon might be result from the rearrangement of small fragments that maked up the A fragment and B fragment due to MGEs; the third segment 105,256 bp to 138,959 bp of p156070-KPC and p158590-KPC were collinear comparison, with a similarity of $100 \%$.

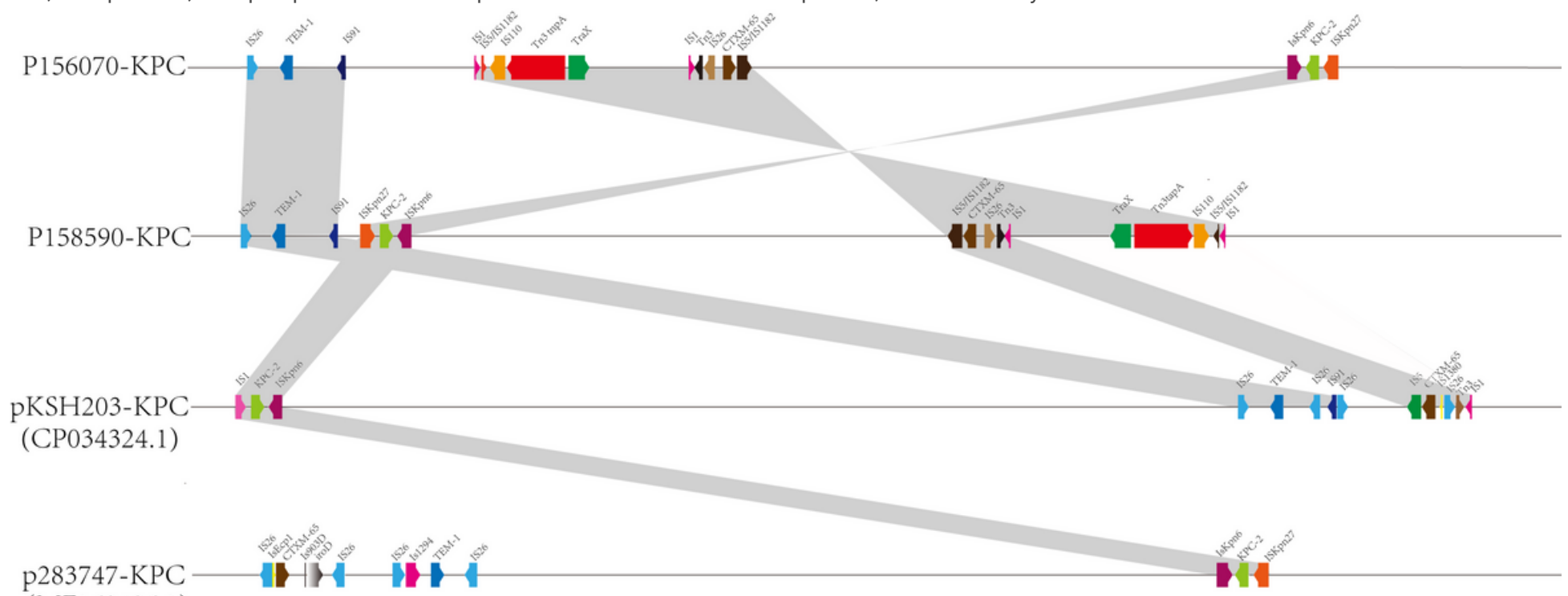

(MF168406.1)

\section{Figure 3}

BLASTN analysis showed that p156070-KPC and p158590-KPC were 96\% similar to the previously reported IncFIl plasmid p283747-KPC (MF168406.1), which harbored blaKPC-2 between ISKpn27 and ISKpn6 
pNFYYOY11

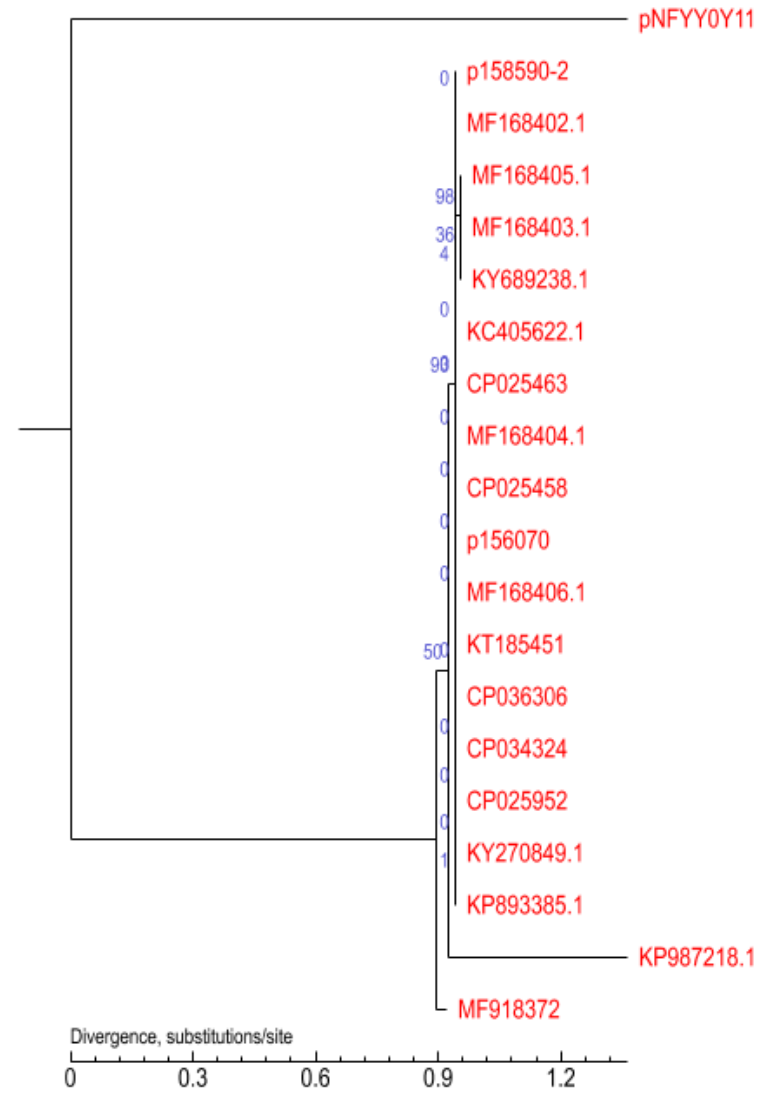

Figure 4

Phylogenetic analysis showed that p156070-KPC and p158590-KPC are closely genetically related to most of the IncFIl plasmids previously reported

\section{Supplementary Files}

This is a list of supplementary files associated with this preprint. Click to download.

- Supplementarytable1.docx

- Supplementarytable2.docx

- Supplementarytable3.docx

- Supplementarytable4.docx

- Supplementarytable5.docx

- SupplementaryFigure1.docx

- SupplementaryFigure2.docx 